\title{
Intensive care unit length of stay beyond the first week and 1-year mortality - dutch single centre study in unselected critically ill patients describing long-term survival according to length of stay in conjunction with age
}

\author{
IW Soliman ${ }^{2 *}$, DW de Lange ${ }^{1}$, LM Peelen², W Pasma ${ }^{1}$, JJM van Delden³ ${ }^{3}$ D van Dijk
}

From ESICM LIVES 2015

Berlin, Germany. 3-7 October 2015

\section{Introduction}

Increasing age and prolonged intensive care unit (ICU) length of stay (LoS) are both associated with in-hospital mortality. Especially older patients with a prolonged ICU stay are often considered to be at high risk for mortality [1]. However the effect of a prolonged ICU LoS in conjunction with age on long-term survival remained to be specified.

\section{Objectives}

We aimed to report one-year survival of ICU patients across different age strata and different LoS strata.

\section{Methods}

All consecutive patients admitted to the ICU of the University Medical Centre Utrecht in the Netherlands between July 2009 and April 2013 were included in the study. For patients with multiple ICU admissions we only included their first admission. Data on patient characteristics were prospectively gathered according to APACHE IV definitions.[2] Survival was tracked using the municipal registry, until one year after ICU admission. Patients were grouped according to age and according to LoS. Note that the LoS groups were not mutually exclusive. The ethics committee of the University Medical Centre Utrecht approved the study and gave a waiver of informed consent (protocol number 10/006).

2Julius Center for Health Sciences and Primary Care University Medical Center Utrecht, Department of Epidemiology, Utrecht, Netherlands Full list of author information is available at the end of the article

\section{Results}

Inclusion and overall survival were shown in figure 1. In total, 7165 unique patients were included. Baseline characteristics are shown in table 1 . One year survival varied markedly, from $82.6 \%$ in patients $<65$ years with ICU LoS $<1$ week, to $22.2 \%$ in patients $>80$ years with ICU LoS $>2$ weeks (figure 2). In all LoS strata, increasing age was associated with a decreasing one year survival. There was also a distinct drop in one year survival when ICU LoS was more than 1 week, in every age category.

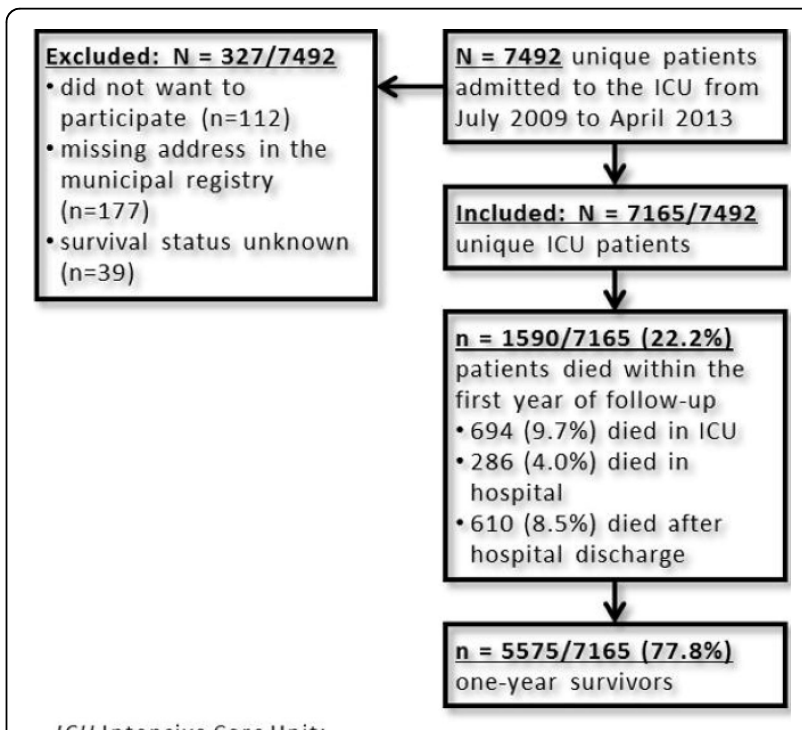

ICU Intensive Care Unit;

Figure 1 Flowchart of inclusion and overall survival. 
Table 1 Baseline characteristics.

\begin{tabular}{cccccc}
\hline & & \multicolumn{2}{c}{ Age at admission } \\
\hline $\mathbf{N}$ & Total population & Under 65 & $\mathbf{6 5 - 7 5}$ & $\mathbf{7 5 - 8 0}$ & $\mathbf{8 0}$ and over \\
\hline Age (years) & 7165 & 3806 & 1938 & 826 & 595 \\
\hline Gender (male) & $64(52-73)$ & $53(43-60)$ & $70(67-72)$ & $77(76-79)$ & $83(81-85)$ \\
\hline ICU length of stay (days) & $4514(63 \%)$ & $2424(63.7 \%)$ & $1254(64.7 \%)$ & $505(61.1 \%)$ & $331(55.6 \%)$ \\
\hline Elective admission & $1(1-3)$ & $1(1-4)$ & $1(1-2)$ & $1(1-2)$ & $1(1-3)$ \\
\hline APACHE IV score & $3908(54.5 \%)$ & $1764(46.3 \%)$ & $1265(65.3 \%)$ & $547(66.2 \%)$ & $332(55.8 \%)$ \\
\hline Total maximum SOFA score* & $46(33-66)$ & $40(27-62)$ & $49(38-65)$ & $52(41-69)$ & $58(46-77)$ \\
\hline
\end{tabular}

Continuous variables are presented as median (interquartile range), categorical variables as $\mathrm{n}$ (percentage); ${ }^{*}$ Sum of highest SOFA component scores during admission. ICU Intensive Care Unit; LoS Length of Stay; APACHE IV Acute Physiology And Chronic Health Evaluation fourth edition; SOFA Sequential Organ Failure Assessment;

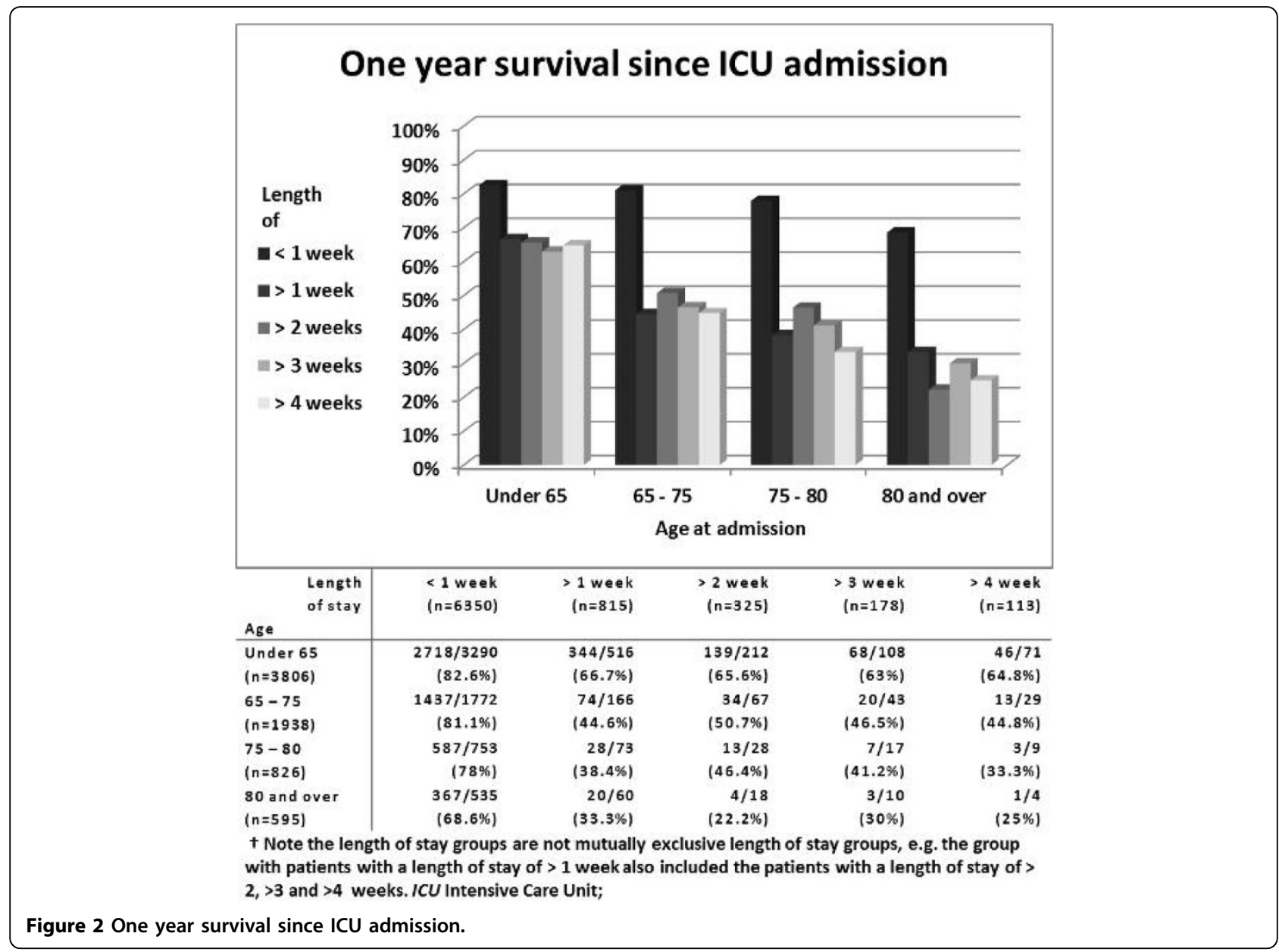

However, after the first week in the ICU, one year survival stayed nearly consistent in patients of all age categories, irrespective of ICU LoS.

\section{Conclusions}

In ICU patients, increasing age was associated higher one year mortality. However, once the patient has survived more than 2, 3 and 4 weeks of ICU stay respectively, his or her 1-year survival is comparable to patients who have been at the ICU for only more than one week.

\section{Grant Acknowledgement}

This study was supported by the NutsOhra Foundation, project nr 1404-013. 


\section{Authors' details}

'University Medical Center Utrecht, Department of Intensive Care, Utrecht, Netherlands. ${ }^{2}$ Julius Center for Health Sciences and Primary Care University Medical Center Utrecht, Department of Epidemiology, Utrecht, Netherlands.

${ }^{3}$ Julius Center for Health Sciences and Primary Care University Medical

Center Utrecht, Department of Medical Humanities, Utrecht, Netherlands.

Published: 1 October 2015

\section{References}

1. Turnbull AE, et al: Age and decisions to limit life support for patients with acute lung injury: a prospective cohort study. Crit Care 2014, 18(3): R107, May 26.

2. Zimmerman JE, et al: Acute Physiology and Chronic Health Evaluation (APACHE) IV: hospital mortality assessment for today's critically ill patients. Crit Care Med 2006, 34(5):1297-310, May.

doi:10.1186/2197-425X-3-S1-A149

Cite this article as: Soliman et al: Intensive care unit length of stay beyond the first week and 1-year mortality - dutch single centre study in unselected critically ill patients describing long-term survival according to length of stay in conjunction with age. Intensive Care Medicine Experimental 2015 3(Suppl 1):A149.

\section{Submit your manuscript to a SpringerOpen ${ }^{\mathcal{O}}$ journal and benefit from:}

- Convenient online submission

- Rigorous peer review

- Immediate publication on acceptance

- Open access: articles freely available online

- High visibility within the field

- Retaining the copyright to your article

Submit your next manuscript at $\gg$ springeropen.com 